\section{Giant inflammatory pseudotumor of the larynx treated endoscopically by a gastroenterologist using submucosal dissection}

Submucosal pedunculated masses of the hypopharynx, larynx, and upper esophagus pose diagnostic and treatment challenges for gastrointestinal endoscopists. Because of potentially acute respiratory complications, the removal of these pedunculated masses through a transoral, transcervical, transthoracic, or endoscopic approach, depending on their location and size, is mandatory [1].

Inflammatory pseudotumors of the larynx are rare, borderline neoplasms of unknown etiology and with uncertain malignant potential that often show locally aggressive behavior [2]. These pedunculated submucosal masses are most commonly approached via endoscopic excision (dissection of the peduncle by ligature, electrocoagulation, or laser), high dose steroids, radiation, or surgical excision [3-5]. We report the case of an 81-year-old man, with significant cardiovascular co-morbidities, who presented with hoarseness, dysphagia, and fatigue that had progressed during the past 4 years. Upper gastrointestinal endoscopy revealed a giant submucosal mass of the right vocal fold originating from the right arytenoid cartilage; the pedicle thickness was $2 \mathrm{~cm}$. Computed tomography showed the mass originating from the larynx ( $\bullet$ Fig.1) without lymph node involvement.

The pedunculated mass was removed endoscopically with submucosal dissection followed by pedicle resection, with a favorable outcome ( $\bullet$ Fig. 2, $\bullet$ Video 1 ). A transparent cap was attached to a gastroscope. Just below the upper esophageal sphincter, submucosal dissection was performed with an Olympus DualKnife (Olympus, Tokyo, Japan), and mucosal hemostasis was achieved with an Olympus Coagrasper. The dissection was done gradually until complete resection of the pedicle had been achieved; the total time for dissection was 112 minutes. No signif-

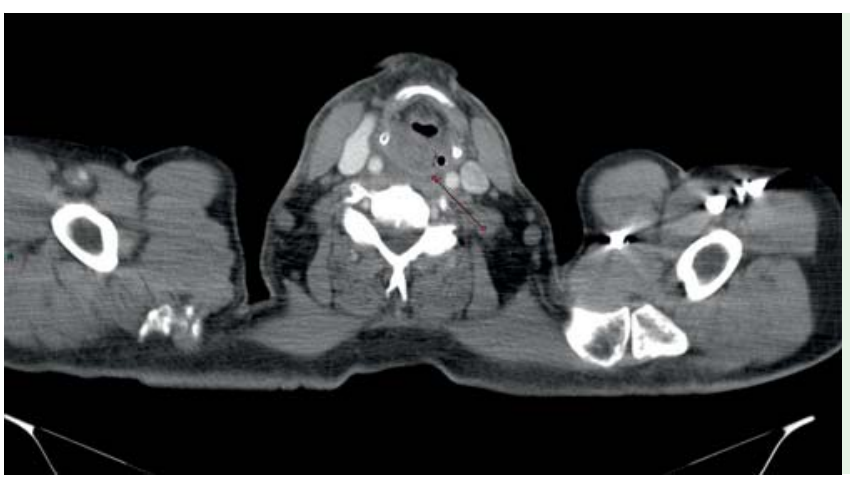

Fig. 1 Computed tomography reveals a mass originating from the larynx, without lymph node involvement, in an 81-year-old man presenting with progressive hoarseness, dysphagia, and fatigue.

icant bleeding or perforation occurred. Two clips were used to close the esophageal mucosal break.

Histological analysis revealed an inflammatory pseudotumor ( $\bullet$ Fig.3). At 6-month follow-up, the patient's clinical status was good, without endoscopic signs of tumor recurrence.

Giant pedunculated submucosal tumor masses of the larynx can be safely removed endoscopically in gastrointestinal endoscopy units with submucosal dissection and pedicle resection.

\title{
Endoscopy_UCTN_Code_TTT_1AO_2AN
}

\section{Competing interests: None}

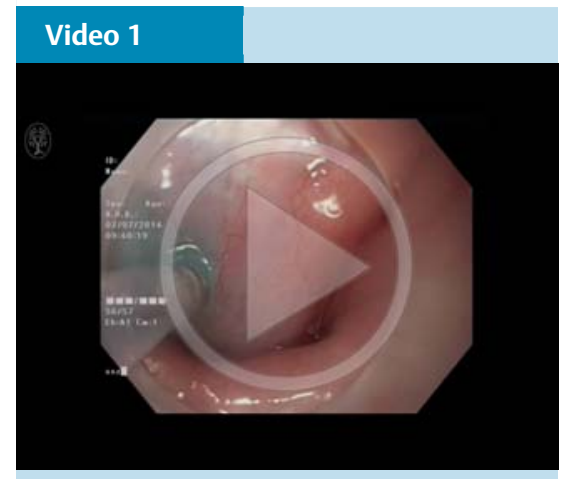

Giant inflammatory laryngeal pseudotumor removed endoscopically. Initial endoscopic inspection revealed the laryngeal giant mass, with a thick pedicle, descending into the esophagus. Submucosal dissection was initiated with a transparent cap just below the upper esophageal sphincter. Mucosal dissection was done with an Olympus DualKnife, and mucosal hemostasis was achieved with an Olympus Coagrasper. The dissection was carried out gradually, layer by layer, until the pedicle had been completely resected. No significant bleeding occurred. Removal from the esophagus was difficult and was possible only after partial fragmentation of the mass.
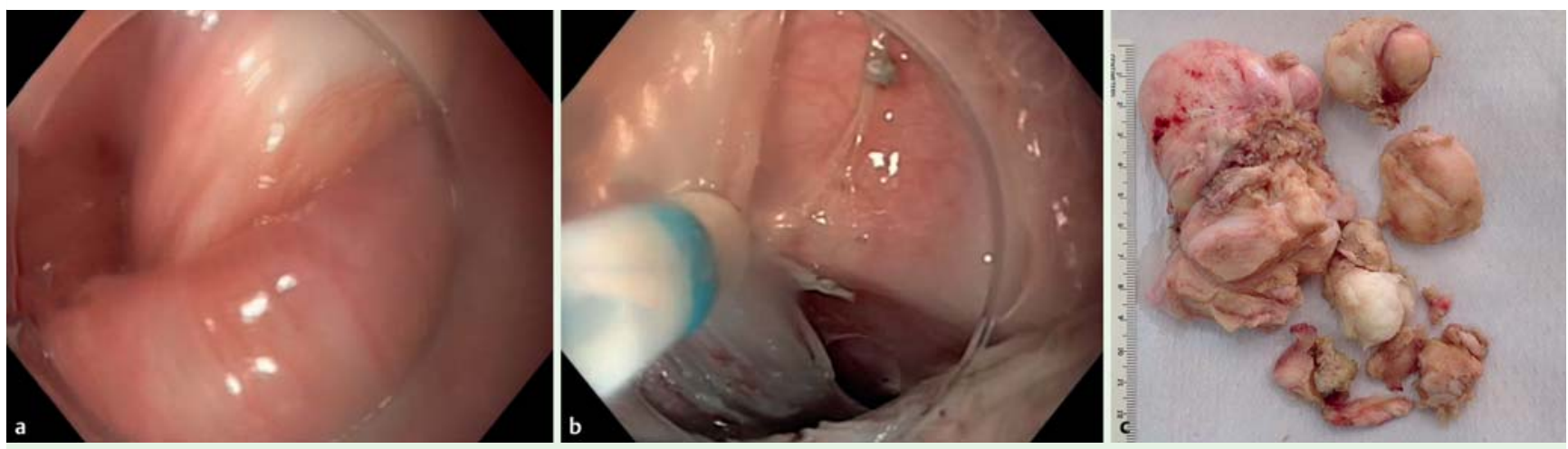

Fig.2 a Pedicle thickness of the laryngeal submucosal pedunculated mass. b Endoscopic dissection of the pedicle. c Macroscopic appearance after endoscopic removal. 


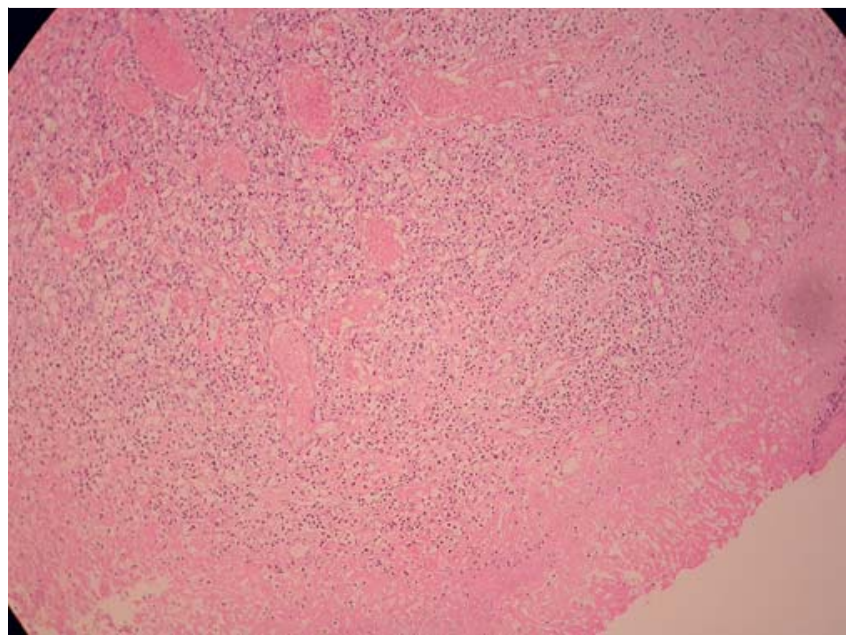

Fig. 3 Microscopic histological analysis (hematoxylin and eosin stain) reveals the presence of mixed inflammatory cells, mainly leukocytes, and fibrovascular areas with hyalinization.

\section{Marcel Tanțău1 ${ }^{1}$, Lidia Ciobanu1 ${ }^{1}$, Teodor Zaharie², Gabriela Mester ${ }^{3}$, Alina Tanțău ${ }^{4}$}

${ }^{1}$ University of Medicine and Pharmacy luliu Hatieganu, Regional Institute of Gastroenterology and Hepatology, Gastroenterology, Cluj-Napoca, Romania

2 Regional Institute of Gastroenterology and Hepatology, Histopathology,

Cluj-Napoca, Romania

${ }^{3}$ Regional Institute of Gastroenterology and Hepatology, Endoscopy,

Cluj-Napoca, Romania
${ }^{4}$ University of Medicine and Pharmacy luliu Hatieganu, Regional Institute of Gastroenterology and Hepatology, 4th Medical Clinic, Cluj-Napoca, Romania

\section{Acknowledgment}

This paper was published with support from the European Social Fund, Human Resources Development Operational Program 2007-2013, project no. POSDRU 159/1.5/S/138776 (TRANSCENT).

\section{References}

1 Pallabazzer G, Santi S, Biagio S et al. Difficult polypectomy-giant hypopharyngeal polyp: case report and literature review. World J Gastroenterol 2013; 19: 5936-5939

2 Do BA, Varshney R, Zawawi $F$ et al. Inflammatory myofibroblastic tumor of the larynx - a case report. J Voice 2014; 28: 258-261

3 Zitsch RP 3rd, Pollak N, Loy TS. Management of inflammatory pseudotumor of the larynx. Otolaryngol Head Neck Surg 2007; 136: 139-141

4 Alobid I, Vilaseca I, Fernández J et al. Giant fibrovascular polyp of the esophagus causing sudden dyspnea: endoscopic treatment. Laryngoscope 2007; 117: 944 -945

5 Zhang J, Hao JY, Li SW et al. Successful endoscopic removal of a giant upper esophageal inflammatory fibrous polyp. World J Gastroenterol 2009; 15: 5236 -5238

\section{Bibliography}

DOI http://dx.doi.org/

10.1055/s-0034-1392503

Endoscopy 2015; 47: E352-E353

(c) Georg Thieme Verlag KG

Stuttgart · New York

ISSN 0013-726X

\section{Corresponding author}

\section{Lidia Ciobanu, MD}

University of Medicine and Pharmacy Iuliu Hatieganu

Regional Institute of Gastroenterology and Hepatology

19-21, Croitorilor Street

Cluj-Napoca, 400162

Romania

Fax: +40-264-455-995

ciobanulidia@yahoo.com 